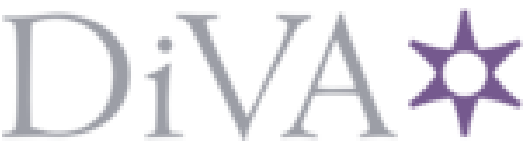

http://www.diva-portal.org

Postprint

This is the accepted version of a paper presented at 2020 Forum for Specification and Design Languages (FDL).

Citation for the original published paper:

Ungureanu, G., Jordao, R., Sander, I. (2020)

Exploiting Dataflow Models for Parallel Simulation of Discrete Timed Systems

In: Kiel, Germany

N.B. When citing this work, cite the original published paper.

Permanent link to this version:

http://urn.kb.se/resolve?urn=urn:nbn:se:kth:diva-28606o 


\title{
Exploiting Dataflow Models for Parallel Simulation of Discrete Timed Systems
}

\author{
George Ungureanu, Rodolfo Jordāo and Ingo Sander School of EECS, KTH \\ Royal Institute of Technology, Stockholm, Sweden \\ Email: \{ugeorge, jordao, ingo\}@kth.se
}

\begin{abstract}
The shift towards parallel computing witnessed since the turn of this century has forced us to rethink traditional software design paradigms to better utilize resources. Yet, the simulation of timeaware systems remains a challenging topic due to the inherent semantics of time and causality whose consistency needs to be controlled, traditionally in form of a global event queue, limiting the potential for parallel exploitation. We propose a rehash of this problem by tackling it from a different modeling perspective, one which is able to express concurrency more naturally, i.e. dataflow (DF) models of computation (MoCs). By abstracting time aspects as an algebra hosted on a pure DF MoC, we are able to apply recent results from $\mathrm{MoC}$ theory not only for the purpose of describing deterministic behaviors for distributed timed systems, but also to overcome the existing limitations of timed execution in order to increase a simulation model's performance. We use a well-known example of a deadlock-prone distributed discrete event system as a driver to introduce the modeling concepts and show their potential for parallelism.
\end{abstract}

\section{Index Terms}

models of computation, parallel simulation, dataflow, discrete event systems

\section{INTRODUCTION}

Cyber-physical system (CPS) design is a relatively new engineering branch that focuses on the integration of physical processes with computation, where both domains exert feedback upon each other. Two of the key concepts in CPS are time, which refers to the causal passage of physical time and concurrency, which refers to the simultaneous occurrence of events. Since the pioneering work of Lee and Sangiovanni-Vincentelli [1], models of computation (MoC) have been used to reason about these two notions along with other extra-functional properties of systems, being capable of formally defining what system behavior means. The nature of CPS requires them to asses the passing of Newtonian time, yet if we want to model or simulate such systems, we depend exclusively on digital computing machines, whose inherent binary encoded nature limits their notion of time to at best a discrete format. To cope with this, algebraic models have been developed and perfected that decompose such continous time models to numerical problems for the purpose of computer execution. The discrete event (DE) model [2] is one such formalization which captures the behavior of timed concurrent systems in a generic, computable manner.

Although DE simulations (DES) are themselves computing intensive software, they are still mainly sequential or expose a limited use of parallelism. This can be attributed to the fact that, in order to maintain determinism, DE imposes a global notion of consistency for the passage of time for all components in a system. This constraint is resolved in practice using a global event queue which, as a shared resource, greatly reduces the potential for parallel exploitation. The more mature discipline of parallel discrete event simulation (PDES) has mainly been focused towards obtaining efficient and consistent distribution of discrete program simulations, usually 


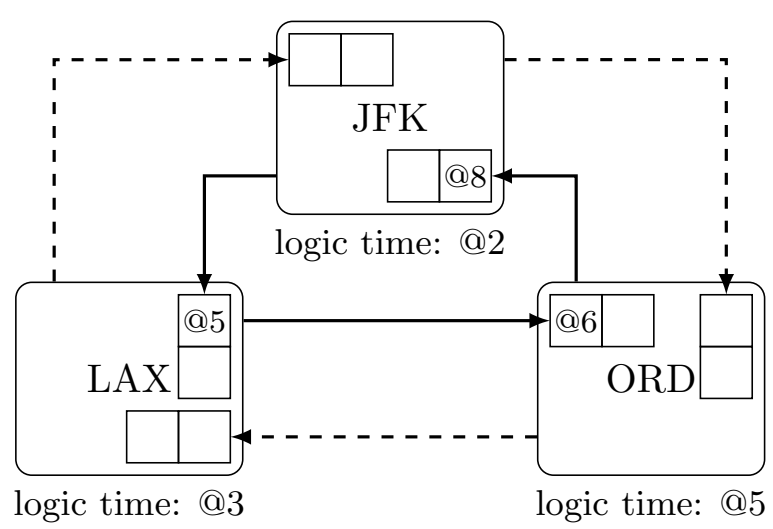

Fig. 1: The 3-Airports Problem

involving trade-offs between generality and performance [3]. Only recently it has acknowledged CPS design, under the term interoperable models [4], and formulated research directions using as examples Ptolemy II and AToM3.

Our goal is to bring the perspective of a formal computational model to DES, as the former is commonly technology-agnostic and may be better suited for parallel execution. Specifically, we argue that a more suitable class of MoCs for natural, non-restrictive distributed execution is the class of dataflow models. We summarize our approach and contributions as follows: 1) we describe a DES model as an algebra hosted in a dataflow model, thus obtaining a conservative PDES engine; 2) we apply both dynamic formulations of dataflow MoCs and simple graph traversal to overcome the drawbacks of unnecessary broadcast control messages, while preserving determinancy. 3) as proof-of-concept we implement this model as an embedded domain specific language (EDSL). We use the host's parallel core and safe evaluation strategies to display wellbalanced multi-threaded execution.

To the best of our knowledge this is the first attempt to consider dataflow MoC techniques for the purpose of PDES.

\section{The Time Challenge}

We highlight the challenge of distributing discrete event simulations through a school-book problem introduced by Fujimoto in [3], which we will use throughout this paper:

1) 3-Airports Problem (3AP): Consider three independent concurrent processes modeling the air traffic control of three airports, JFK, LAX and ORD. They are interconnected like in Fig. 1. Each traffic controller has a notion of (local) logic time (LT) that can only advance incrementally. Assuming that a plane takes 3 time units to travel between any two airports, whenever a new outbound flight occurs, the source controller sends the destination controller a message with the arrival time. To maintain simulation consistency, no airport controller is allowed to "peak into the future", meaning that each LT can be incremented at most to a value that is guaranteed to be lower than any possible future input timestamp. Consider the following scenario: after some initial activity the controller LTs are @2, @3 and @5 respectively. At these LTs JFK sends an outbound flight message to LAX, LAX to ORD and ORD to JFK, as depicted in Fig. 1. At this instant ORD cannot process the token in the LAX input queue and advance its logical time from@5 to@6 because it has no inputs from JFK to reason its current time, hence there is no guarantee that further messages from JFK will have timestamp lower than @6. The other logical controllers are in this same situation. As a result, this system simulation deadlocks and cannot advance. 
2) Deadlock. Zeno Behavior: The previous phenomenon can be explained from the perspective of the tagged signal model [1]. Within this theoretical framework, systems are regarded as compositions of processes acting on signals. A signal defines a behavior and is a set of tagged events where each event is composed of a tag and a value, and tag systems are determining MoCs. If the tag system is totally ordered, then we say the MoC is timed, and the signals admit a metric space. This enables the definition of precise notions of causality in systems [1]. Zeno behavior occurs in (discrete) timed feedback systems which are both 1) non-strictly causal and; 2) non-delta causal, i.e. not characterized by a contraction mapping. In this case the system oscillates indefinitely but is never able to advance model time. Zeno behavior would have been observed in the $3 A P$ if the airports would not have the pre-knowledge of a flight duration: in that case no matter how many messages are exchanged all LTs would be stuck at the same timestamp.

If a tag system is partially ordered, then we say the $\mathrm{MoC}$ is untimed. In this case causality is measured in terms of sequential consistency on a partial metric of signal prefixes. Deadlock occurs in untimed feedback systems which are either: 1) non-monotonous; or 2) do not define a prefix behavior over the feedback signal. In this case the only possible system behavior is inaction [1]. The $3 A P$ is displaying in fact a deadlock, judging that each airport is in fact an (untimed) computing station: the dotted feedback loop lacks a prefix behavior.

3) Discrete Event Simulation: At the highest level of abstraction, a DES can be modeled as a timed automaton [5] where a clock structure dictates which event needs to be processed at each tag. The state of the automaton advances in deterministic fashion only if there is no event to be processed at a certain tag [2, Ch. 10]. Nonetheless, to compute such a behavior, we must maintain a scheduled event list ordered in ascending time order, i.e. the first event in the list is always the triggering event. This implies that for each triggering of an event, after updating the new state and time, the list needs to be parsed and updated by deleting infeasible events, adding new feasible events and reordering it accordingly. To guarantee consistency this list becomes a mandatory bottleneck, crippling any attempt for substantial parallelization.

PDES literature identifies two major classes of approaches for distribution of DES onto socalled logical processes (LP): conservative where LPs work independently but are restricted to process events in timestamp order; and 2) optimistic where LPs can execute ahead of time but require complex rollback mechanisms in case causality is violated. For the scope of this work we consider conservative approaches due to their technology agnosticism. The first conservative algorithm (CMB) was developed by Chandy, Misra and Bryant [6] and assumes that each LP can guarantee its neighbors that it will never send an event with a smaller timestamp than its LT @ $t$. This is done by sending a NULL message with timestamp @ $t+l$ where $l$ is a mandatory lookahead value modeling the channel delay. Consider the 3AP: if JFK sends NULL@2+3, ORD understands it is safe to increment its LT @6; the process is repeated and system time always advances. However, this algorithm suffers from a phenomenon called lookahead creep: assume $l$ is 0.1 instead of 3 , then the airports need to exchange NULL messages every 0.1 units until ORD can decide to process the event @6, generating significant synchronization overhead. Subsequent conservative algorithms have addressed this issue, mainly by calculating synchronization cycles for safe concurrent processing yet all depend on a generous lookahead $l$ in order to leverage enough parallelism. Contemporary approaches that augment the principle of conservative execution include Ptides [7] and Google Spanner [8].

\section{DATA-Driven TIMED Simulation}

Our hypothesis is that if we can describe a DES as a dataflow problem, we are able to better leverage the potential for distribution of simulation workload, thus tailoring an efficient resource 


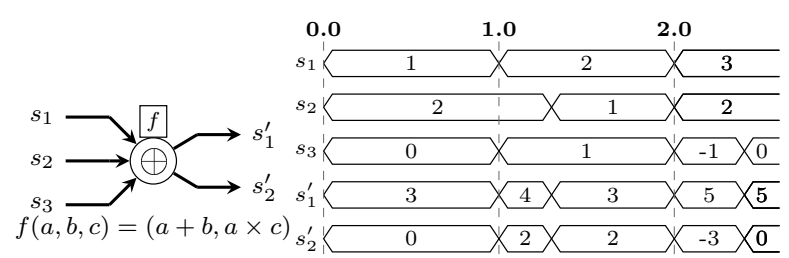

Fig. 2: Example of $f \oplus\left(s_{1}, s_{2}, s_{3}\right)=\left(s_{1}^{\prime}, s_{2}^{\prime}\right)$

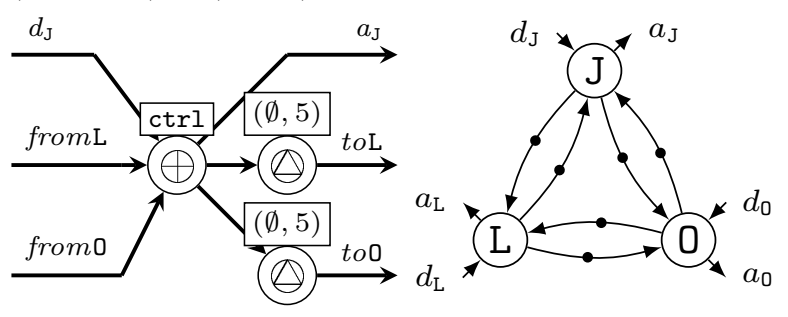

Fig. 4: 3-Airports Problem model

utilization with easier parallelization. This is possible since the scheduling and evaluation strategies rely solely on data dependencies, whereas the job of causality falls on each actor performing well-defined time arithmetics.

First we distinguish between two modeling paradigms with orthogonal purposes: 1) a host dataflow model which grants the execution semantics and evaluation strategies; and 2) an embedded language of interactions, enabling the modeling and simulation of timed behavior. As formal framework for the host we describe a dataflow MoC in terms of pure functional programs on (infinite) lists [9], also called streams [10]. Lists are recursive algebraic structures capable of modeling and deriving an evaluation semantics for sequences, and have been at the foundation of dataflow languages [10]. For embedding the $\mathrm{DE} \mathrm{MoC} \mathrm{semantics,} \mathrm{we} \mathrm{use} \mathrm{the} \mathrm{concept} \mathrm{of} \mathrm{process}$ constructors [11] over streams. Our idea is thus to transliterate the tagged signal model [1] into such a functional interpretation, define an appropriate tag system, and describe a minimal set of causal mappings over it in terms of process constructors, in order to simulate deterministic timed models.

Let the recursive structure $S(\alpha)=\alpha_{@ \tau} \prec S(\alpha)$ define a stream, where each element is a value $\in \alpha$ associated with a discrete tag $\in \tau$, used to model a DE signal. We define the combinational process constructor $\oplus$ such that $\forall\left(v_{i @ t_{i}} \prec \ldots\right), i \in 1 . . n$ being its input signals, it synchronizes all signals and triggers an evaluation $f\left(v_{1}, \ldots v_{n}\right)_{@}$ where $t=\min \left(t_{i}\right)$, after which it discards the triggering events $@ t$, as shown in Fig. 2. The delay process constructor is defined such that $\left(x_{@ 0} \prec \ldots @ d \prec \ldots\right) \oplus s=x_{@ 0} \prec \operatorname{map}\left(v_{@ t} \mapsto v_{@ t+d}\right) s$, meaning that it infers a value $x$ and a duration $d$ from the left-hand signal and sets the prefix, respectively increments all tags in the right-hand with $d$. For brevity we also use the notation $(x, d) \otimes s$. The $\oplus$ process constructor ensures monotonicity for any arbitrary $f$, respectively $\oplus$ ensures both prefix behavior and strict causality for any arbitrary composition. Thus it can be proven that any feedback composition using these two constructors, is Zeno-free and deadlock-free, e.g. as shown in Fig 3. The monotonicity induces an overly-conservative behavior, hence it can also be proven that this model is in fact a dataflow implementation of the CMB algorithm.

1) Modeling the 3-Airports Problem: Let the type $\mathcal{F}=$ Flight $\cup \emptyset$ where $\emptyset$ represents a NULL message, and a Flight is a structure (src, dest, num) carrying flight information. The airports are modeled in terms of $\oplus$ and $\oslash$ processes operating on signals $\in S(\mathcal{F})$, like in Fig. 4. For brevity 
we have annotated the airports with their initials, i.e. J for JFK, etc. For the didactic purpose, we consider a simple scenario: we disregard landing times, landing queues or flight re-departures. For example, for the JFK controller, the process function $\operatorname{ctrl}\left(s_{\mathrm{J}}, f_{\mathrm{L}}, f_{\mathrm{0}}\right)=\left(\operatorname{land}\left(f_{\mathrm{L}}, f_{\mathrm{O}}\right), \operatorname{dept}\left(s_{\mathrm{J}}\right)\right)$ is demultiplexing conservatively (i.e. using $\emptyset$ ) the scheduled outbound flights received through signal $d_{\mathrm{J}}$ using the dept rule; respectively monotonically syncing inbound flights and placing them into the $a_{\mathrm{J}}$ signal using the land rule. The outbound flights are passing through a $\otimes$ process modeling the flight duration, but also keeping the circular composition causal. The remaining two airports are instantiated similarly, and composing all of them together yields an implementation of the $3 A P$ system in Fig. 4, denoted with the acronym 3AP-CMB. We have implemented this system in the Haskell language and, as expected, obtained the behavior in Fig. 6a. Notice the lookahead creep phenomenon typical to the CMB algorithm.

2) Parallel Strategies: We briefly demonstrate the proof-of-concept EDSL, by making use of a dynamic thread scheduling model based on the concept of "sparks", implemented in the Haskell parallel core [12]. A spark is an un-evaluated expression which can potentially be assigned to execute in parallel by a run-time scheduler (RTS) if resources are available and data dependencies are met. The creation of sparks is controlled with the operator $\|$ defined below, which forces evaluation of a signal sig, in parallel (par) with an arbitrary expression exp. The forceseq function dictates that each event token in a stream is to be evaluated to normal form ( $r \mathrm{f}$ ), in sequence (pseq). If a safe-lookahead methodology exists, e.g. [7], forceSeq could be improved to make use of it.

sig $\| \exp =$ (forceSeq sig) 'par' exp

forceSeq $(s \prec s s)=$ (rnf $s$ ) 'pseq' forceSeq $s s$

3AP-CMB-par evaluate each airport output in parallel while the program computes the landing signals by declaring $a_{\mathrm{J}}\left\|a_{\mathrm{L}}\right\| a_{\mathrm{O}} \|\left(a_{\mathrm{J}}, a_{\mathrm{L}}, a_{\mathrm{0}}\right)$. Fig. 7 profiles a random-generated simulation of $10^{3}$ t.u. (time units) out of which approx. 16\% are events, on an Intel i7-3770 desktop. Comparing the workload of a sequential 3AP-CMB on one core against the parallel 3AP-CMB-par on three cores, we notice that the implementation is $4.2 \mathrm{x}$ slower in the parallel version $(\approx 0.59 \mathrm{~s})$ as compared to the original sequential version $(\approx 0.14 \mathrm{~s})$. Remark: Haskell was used for fast prototyping due to its innate support for dataflow semantics as pure functions on immutable objects (streams). However the run-time penalty associated with its implicit mechanisms, e.g. garbage collecting (GC) intermediate objects such as unevaluated sparks, is much higher than for explicit resource management [12]. Yet the profiles in Fig. 7 give a good "feeling" of the dynamics of the $3 A P$ system: the utilization inside each process is far too insignificant as compared to the (thread) management for conservative synchronization.

3) Exploiting dynamic dataflow MoCs: The proposed PDES language, although deterministic and well-behaved, suffers the same drawbacks as the first generation of conservative PDES, namely the lookahead creep. To overcome this, we need to admit non-conservative behaviors, i.e. the possibility to shut down/turn on channels, yet to still benefit from liveness analysis methods. For this type of behavior we consider the scenario-aware DF (SADF) and Boolean DF (BDF) MoCs.

a) SADF Approach: The SADF MoC is a generalization of SDF which captures both the dataflow processing part and the control part responsible for determining various scenarios [13]. SADF identifies two types of actors: kernels, which are regular processes and detectors, which are state machines observing the state of the process network by reading data signals, and capable of modifying the kernels' behavior. As such, SADF kernels can effectively close channels, while still benefiting from liveness and boundness analysis [13].

We extend our original three airports system to the 3AP-SADF represented in Fig. 5a. A detector $D$ monitors the status of all three departure channels, and controls the operation mode of each 


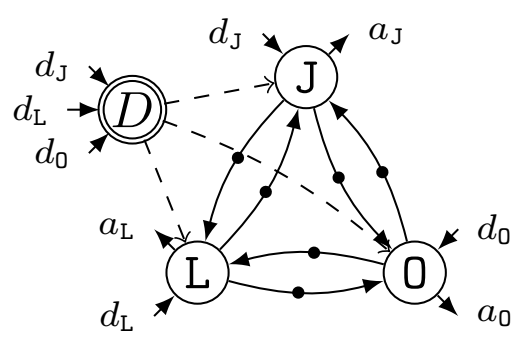

(a) 3AP-SADF

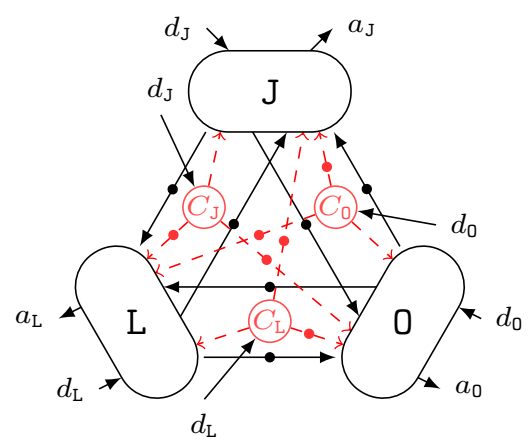

(b) 3AP-BDF

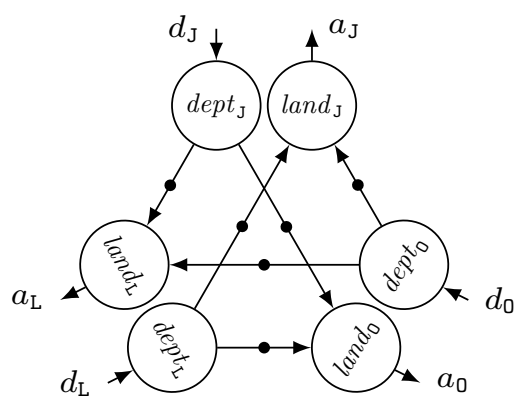

(c) 3AP-DP-OPT

Fig. 5: Alternative dynamic DF designs

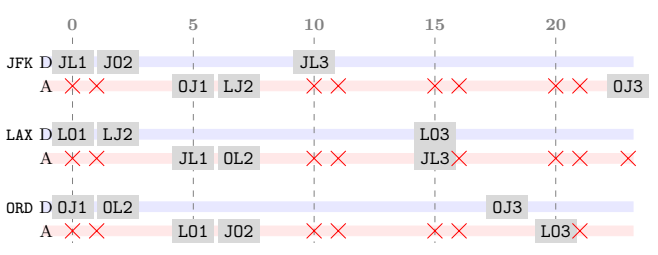

(a) Original 3AP-CMB design

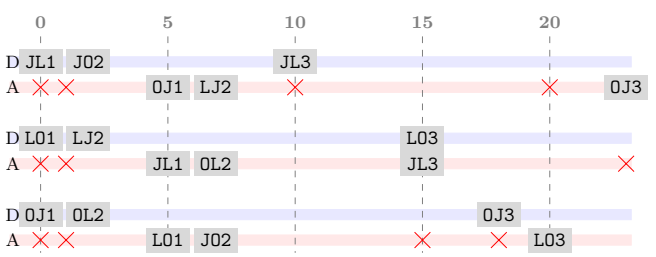

(b) 3AP-BDF design

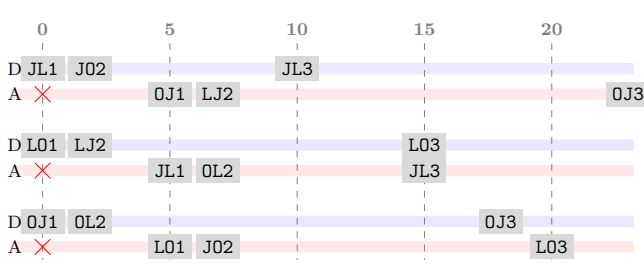

(c) 3AP-DF-OPT design

Fig. 6: Example execution: D = departures; A $=$ arrivals; SrcDstNum = flights; $\times=$ NULL messages; flight duration $=5$ t.u.
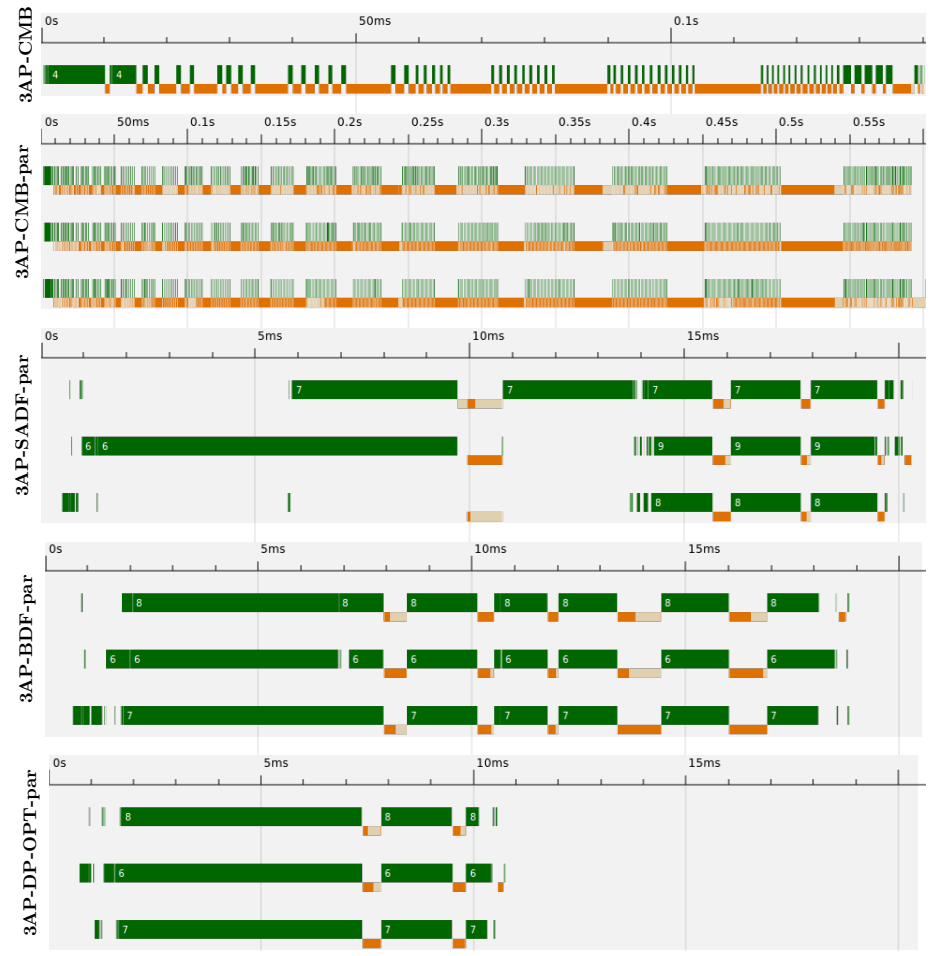

Fig. 7: Workload distribution for random simulation of $10^{3}$ t.u. $\square=$ running; $\square=\mathrm{GC} ; \square=\mathrm{GC}$ waiting

airport, modeled now as kernels. The detector operates conservatively, yet the lookahead creep between the airports is completely avoided. In turn we gain a speedup of $7 \mathrm{x}(\approx 21 \mathrm{~ms})$ for running 3AP-SADF-par as compared to the original 3AP-CMB design, as seen in Fig. 7. Using a detector is different than using a dedicated global event queue process because: 1) all processes are monotonic, i.e. there is no event reordering; 2) consistency needs to be monitored only in feedback loops, also called in literature "cyclic islands" [3].

b) BDF Approach: BDF [14] is an early attempt to raise the expressiveness of SDF by allowing data-dependent firing rules. It defines additional switch and select actors as means to control the redirecting/merging of the data flow in a graph. Due to dynamism BDF loses much 
of the static analysis potential, however in closed-form (e.g. fork-join pattern) it is possible to determine deadlock conditions and bounded cyclic schedules.

Our approach to design 3AP-BDF is based around deriving well-behaved fork-join patterns to model the communication on the send-receive links in the 3AP model. E.g, in Fig. 5b the control process $C_{\mathrm{J}}$ decodes the departure schedule $d_{\mathrm{J}}$ into the Boolean signals which control the switch behavior of $\operatorname{dept}_{\mathrm{J}}$, respectively the select behavior of $\mathrm{land}_{\mathrm{L}}$ and $\mathrm{land}_{\mathrm{O}}$. The example run-time behavior in Fig. 6b shows that lookahead creep is non-existent. The main difference as compared to 3AP-SADF is that the detection control is done per channel instead of per loop. While this increases the parallel processor utilization, see Fig. 7 with a slightly higher speedup of $7.4 \mathrm{x}(\approx 19 \mathrm{~ms})$, the model derivation is rather non-intuitive and problem-specific.

c) Datapath Traversal: The last approach is the most obvious, yet easy to overlook: traverse the data dependecies to identify further potential for parallelization. In the 3AP example, the functions land and dept are independent from each other. Exploiting this information, 3AP-DFOPT can be decomposed into three parallel datapaths. Fig. 6c shows that there is no need for any NULL message, and the application still behaves correctly. Moreover, the workload distribution in Fig. 7 shows the evaluation of the program in three threads independent from each other, hence achieving the maximum achievable speedup for this application: almost $13 \mathrm{x}(\approx 11 \mathrm{~ms})$.

\section{CONCLUSIONS}

We advocate that off-line analysis of an application model is capable of deriving better simulation models, tailored for better on-line performance. In this respect we present an algebra of tags hosted on a dataflow model behaving like a conservative general-purpose parallel discrete event simulator. By applying different formulations of dataflow that capture dynamic behaviors such as SADF and BDF, we exploit knowledge of the functional specification in order to derive better-suited, fully distributed, deterministic simulation models. As proof-of-concept we implement this model as a DSL embedded in the functional language Haskell and show that it respects DE causality while the different approaches display a palpable increase in parallel utilization and speedup.

Acknowledgement: This research was partially funded by the Swedish Governmental Agency for Innovation Systems, NFFP7 project: Correct by construction design methodology \#2017-04892.

\section{REFERENCES}

[1] E. Lee and A. Sangiovanni-Vincentelli, "A framework for comparing models of computation," IEEE J. Technol. Comput. Aided Design, vol. 17, no. 12, Dec. 1998.

[2] C. G. Cassandras and S. Lafortune, Introduction to discrete event systems. Springer Science \& Business Media, 2009.

[3] R. M. Fujimoto, Parallel and distributed simulation systems. Wiley New York, 2000, vol. 300.

[4] R. Fujimoto, "Parallel and distributed simulation," in Winter Simulation Conference (WSC), 2015. IEEE, 2015 , pp. 45-59.

[5] R. Alur and D. L. Dill, "A theory of timed automata," Theoretical Computer Science, vol. 126, pp. 183-235, 1994.

[6] K. M. Chandy and J. Misra, "Asynchronous distributed simulation via a sequence of parallel computations," Communications of the ACM, vol. 24, no. 4, pp. 198-206, 1981.

[7] J. Zou et al., "Execution strategies for PTIDES, a programming model for distributed embedded systems," in 2009 15th IEEE Real-Time and Embedded Technology and Applications Symposium, 42009.

[8] J. C. Corbett et al., "Spanner: Google's globally distributed database," ACM TOCS, vol. 31, no. 3, pp. 1-22, 2013.

[9] R. S. Bird, An introduction to the theory of lists. Springer, 1987.

[10] G. Kahn, "The semantics of a simple language for parallel programming," Information processing, vol. 74, pp. 471-475, 1974.

[11] I. Sander and A. Jantsch, "System modeling and transformational design refinement in ForSyDe," IEEE J. Technol. Comput. Aided Design, vol. 23, no. 1, pp. 17-32, January 2004.

[12] S. Marlow, "Parallel and concurrent programming in Haskell," in CEFP 2011, ser. LNCS, V. Zsók, Z. Horváth, and R. Plasmeijer, Eds., 2012, vol. 7241, pp. 339-401.

[13] S. Stuijk et al., "Scenario-aware dataflow: Modeling, analysis and implementation of dynamic applications," in 2011 Intl. Conf. on Emb. Comp. Sys.: Architectures, Modeling and Simulation, 72011.

[14] J. Buck and E. Lee, "Scheduling dynamic dataflow graphs with bounded memory using the token flow model," in IEEE International Conference on Acoustics Speech and Signal Processing, - 1993. 